\title{
Differential distributions in rare four-leptonic B-decays.
}

\author{
Anna Danilina ${ }^{1,2,3, *}$ and Nikolay Nikitin ${ }^{1,2,3, * *}$ \\ ${ }^{1}$ Faculty of Physics, M.V. Lomonosov Moscow State University, Moscow, 119991 Russia \\ ${ }^{2}$ D. V. Skobeltsyn Institute of Nuclear Physics, M.V. Lomonosov Moscow State University, Moscow, \\ 119991 Russia \\ ${ }^{3}$ A.I. Alikhanov Institute for Theoretical and Experimental Physics, National Research Center Kurcha- \\ tov Institute, Bol'shaya Cheremuskinskaya ul. 25, Moscow, 117218 Russia.
}

\begin{abstract}
The branching ratios and differential distributions for the fourleptonic decays $B^{-} \rightarrow \mu^{+} \mu^{-} \bar{v}_{e} e^{-}, B^{-} \rightarrow e^{+} e^{-} \bar{v}_{\mu} \mu^{-}$and $B^{-} \rightarrow \mu^{+} \bar{v}_{\mu} \mu^{-} \mu^{-}$are calculated within the Standard Model. The contributions of the virtual photon emission from the $\mathrm{b}$ - and $\mathrm{u}$ - quarks and the bremsstrahlung contribution were taken into account. The resonances contributions were calculated using the vector dominance model. In this paper we present the differential distributions for the $B^{-} \rightarrow \mu^{+} \mu^{-} \bar{v}_{e} e^{-}$decay obtained by two methods. Testing the lepton universality in rare multileptonic decays of charged and neutral B mesons are discussed.
\end{abstract}

\section{Introduction}

Investigation of four-leptonic decays of $B$ - mesons open the possibility for precision tests of Standard Model predictions in higher orders of perturbation theory. Decays of this type could be background processes for the extremely rare helicity suppressed $B_{d, s} \rightarrow \mu^{+} \mu^{-}$decays, that have been the subject of vigorous studies at the Large Hadron Collider (LHC,CERN) [1-3] in connection with searches for physics beyond the Standard Model. The correct simulation is of great importrance for the search of such effects in the rare $B$ - mesons. In this paper we present the theoretical predictions for the decays of charged $B$ - mesons to three charged leptons and neutrino. This article continues the series of articles aimed at $B$ - mesons investigations $[4,5]$. In Sections 2 we consider the effective Hamiltonian and the diagrams contributing to the decays. Sections 3 and 4 represents numerical results for branching ratios, differential distributions and the forward-backward asymmetry of $B$ - mesons four- leptonic decays obtained by two approaches. In one of them the EvtGen Monte Carlo generator [6] based on the BTOMUMUENU model is used. This model can be used directly at the LHC and other simulation procedure. Section 5 is our conclusions.

\footnotetext{
*e-mail: anna.danilina@cern.ch

**e-mail: Nikolai.Nikitine@cern.ch
} 


\section{Theoretical review of the $B^{-} \rightarrow \ell^{+} \ell^{-} \bar{v}_{\ell^{\prime}} \ell^{\prime-}$ decay}

\subsection{Effective Hamltonian}

The Hamiltonian for the $B^{-} \rightarrow \ell^{+} \ell^{-} \bar{v}_{\ell^{\prime}} \ell^{\prime-}$ four-leptonic decays in the terms of the fundamental fields can be represented in the form

$$
\mathcal{H}_{\text {eff }}(x)=\mathcal{H}_{W}(x)+\mathcal{H}_{e m}(x) .
$$

The Hamiltonian for the $b \rightarrow u W^{-} \rightarrow u \ell^{-} \bar{v}_{\ell}$ transitions is given by

$$
\mathcal{H}_{W}(x)=\frac{G_{F}}{\sqrt{2}} V_{u b}\left(\bar{u}(x) \gamma^{\mu}\left(1-\gamma^{5}\right) b(x)\right)\left(\bar{\ell}(x) \gamma_{\mu}\left(1-\gamma^{5}\right) v_{\ell}(x)\right)+\text { h.c. }
$$

where $u(x)$ and $b(x)$ are quark fields, $\ell(x)$ and $v_{\ell}(x)$ are lepton fields, $G_{F}$ is the Fermi constant, $V_{u b}$ is an element of the Cabibbo-Kobayashi-Maskawa matrix, and the matrix $\gamma^{5}$ is defined as $\gamma^{5}=i \gamma^{0} \gamma^{1} \gamma^{2} \gamma^{3}$.

The Hamiltonian for electromagnetic interaction has the form

$$
\mathcal{H}_{\text {em }}(x)=e \sum_{f} Q_{f}\left(\bar{f}(x) \gamma^{\mu} f(x)\right) A_{\mu}(x),
$$

where the elementary charge $e=|e|$ is normalized by the condition $e^{2}=4 \pi \alpha_{e m}$ and $\alpha_{e m} \approx 1 / 137$ being the fine-structure constant, $Q_{f}$ is the charge of the fermion of flavour $f$ in elementary-charge units; $f(x)$ is the fermion field with flavour $f$ and $A_{\mu}(x)$ is the electromagnetic field.

To estimate the intermediate contribution of the virtual vector mesons we use effective the Hamiltonian describing the Vector Meson Dominance (VMD) model in the form

$$
\mathcal{H}_{V M D}(x)=\sum_{V_{i}} \frac{e M_{V_{i}}^{2}}{f_{V_{i}}} V_{i}^{\mu}(x) A_{\mu}(x),
$$

where $V_{i}^{\mu}(x)$ is the field of a vector meson and $M_{V_{i}}$ is its mass. The absolute values of the dimensionless coupling constants $f_{V_{i}}$ for $M_{V_{i}} \gg m_{\ell}$ are calculated on the basis of experimental values $V_{i} \rightarrow \ell^{+} \ell^{-}$branching ratios according to the relation

$$
\Gamma\left(V_{i} \rightarrow \ell^{+} \ell^{-}\right)=\frac{4 \pi}{3}\left(\frac{\alpha_{e m}}{\left|f_{V_{i}}\right|}\right)^{2} M_{V_{i}} .
$$

The coupling constants of $\rho^{0}$ - and $\omega$ - mesons are $f_{\rho}=5.04$ and $f_{\omega}=17.1$ [7].

\subsection{Structure of the amplitudes in the approximation of massless leptons for the $B^{-} \rightarrow \ell^{+} \ell^{-} \bar{v}_{\ell^{\prime}} \ell^{\prime-}$ decay}

In describing $B^{-}(p) \rightarrow \ell^{+}\left(k_{1}\right) \ell^{-}\left(k_{2}\right) \bar{v}_{\ell^{\prime}}\left(k_{3}\right) \ell^{\prime-}\left(k_{4}\right)$ decays there are three types diagrams: a virtual photon is emitted from $u$ quark (see Fig. 1 (a)); a virtual photon is emitted from $b$ quark (see Fig. 1 (b)) and bremsstrahlung diagrams in which a virtual photon is described by the lepton $\ell^{\prime-}$ (see Fig. 1 (c)).

Let us consider the decay processes $B^{-} \rightarrow \mu^{+} \mu^{-} \bar{v}_{e} e^{-}$or $B^{-} \rightarrow e^{+} e^{-} \bar{v}_{\mu} \mu^{-}$with different flavors of the negatively charged leptons in the final state. In a general form, these decays can be represented as $B^{-} \rightarrow \ell^{+} \ell^{-} \bar{v}_{\ell^{\prime}} \ell^{\prime-}$ where $\ell \neq \ell^{\prime}$. We denote $q=k_{1}+k_{2}$ and $k=k_{3}+k_{4}$. The contribution of the diagram in Fig. 1 (a) to the total amplitude for the $B^{-} \rightarrow \ell^{+} \ell^{-} \bar{v}_{\ell^{\prime}} \ell^{\prime-}$ 
decay can be calculated in the approximation of the VMD model. If set $m_{\ell}=m_{\ell^{\prime}}=0$ and use the expression for the effective Hamiltonian, it can be found that Fig. 1 (a) described by:

$$
\mathcal{M}_{f i}^{(u)}=\mathcal{A} \frac{1}{q^{2}}\left[\sum_{i=\rho^{0}, \omega} \frac{M_{2 i}^{2}}{f_{V_{i}}} \frac{1}{q^{2}-M_{2 i}^{2}+i \Gamma_{2 i} M_{2 i}} \mathcal{F}_{\mu \nu}^{(i)}\left(k^{2}\right)\right] j^{v}\left(k_{2}, k_{1}\right) J^{\mu}\left(k_{4}, k_{3}\right),
$$

where, with allowance for the equations of motion, we have

$$
\mathcal{F}_{\mu \nu}^{(i)}\left(k^{2}\right)=\frac{2 V^{(i)}\left(k^{2}\right)}{M_{1}+M_{2 i}} \epsilon_{\mu v k q}-i\left(M_{1}+M_{2 i}\right) A_{1}^{(i)}\left(k^{2}\right) g_{\mu \nu}+2 i \frac{A_{2}^{(i)}\left(k^{2}\right)}{M_{1}+M_{2 i}} q_{\mu} k_{v},
$$

where $M_{1}$ is the mass of $B$ - meson. According to VMD we taking into account only the contributions of the lightest vector mesons $\rho^{0}$ and $\omega$ containing a $u \bar{u}$ pair.

The contribution of the process in the Fig. 1 (b) is the crossing channel of the $B^{*} \rightarrow B \gamma^{*}$ decay of a heavy vector meson to a heavy pseudoscalar meson and a virtual photon:

$$
\mathcal{M}_{f i}^{(b)}=\frac{2 \mathcal{A}}{3} \frac{1}{q^{2}} \frac{M_{B^{*}} f_{B^{*}}}{k^{2}-M_{B^{*}}^{2}+i \Gamma_{B^{*}} M_{B^{*}}} \frac{V_{b}\left(q^{2}\right)}{M_{1}+M_{B^{*}}} \epsilon_{\mu v k q} j^{v}\left(k_{2}, k_{1}\right) J^{\mu}\left(k_{4}, k_{3}\right) .
$$

Parametrizations of the hadron form factors were constructed in [8]. The form factor $V_{b}\left(q^{2}\right)$ calculated within the dispersion formulation of the quark model can be obtained in the form $[9,10]$ :

$$
V_{b}\left(q^{2}\right)=\frac{1.044}{\left(1-\frac{q^{2}}{M_{\Upsilon}^{2}}\right)\left(1-0.81 \frac{q^{2}}{M_{\Upsilon}^{2}}\right)},
$$

where $M_{\Upsilon}$ is the $\Upsilon(1 S)$-meson mass. One can use the same method for the calculation of values of leptonic constants: $f_{B_{u}}=191 \mathrm{MeV}$ and $f_{B^{*}}=183 \mathrm{MeV}$.

The contribution corresponding to Fig. 1 (c) from bremsstrachlung is described with

$$
\mathcal{M}_{f i}^{(\text {brem })}=\mathcal{A} \frac{i f_{B_{u}}}{q^{2}} g_{\mu \nu} j^{\nu}\left(k_{2}, k_{1}\right) J^{\mu}\left(k_{4}, k_{3}\right) \text {. }
$$

In expressions (3), (4) and (6) we employed the following conventions:

$$
\begin{gathered}
\mathcal{A}=-\frac{G_{F}}{\sqrt{2}} 4 \pi \alpha_{e m} V_{u b}, \\
j^{\nu}\left(k_{2}, k_{1}\right)=\left(\bar{\ell}\left(k_{2}\right) \gamma^{\nu} \ell\left(-k_{1}\right)\right) \text { and } J^{\mu}\left(k_{4}, k_{3}\right)=\left(\overline{\ell^{\prime}}\left(k_{4}\right) \gamma^{\mu}\left(1-\gamma^{5}\right) v_{\ell^{\prime}}\left(-k_{3}\right)\right) .
\end{gathered}
$$

The total amplitude for the $B^{-}(p) \rightarrow \ell^{+} \ell^{-} \bar{v}_{\ell^{\prime}} \ell^{\prime-}$ decay has the form

$$
\begin{gathered}
\mathcal{M}_{f i}^{(1234)}=\mathcal{M}_{f i}^{(u)}+\mathcal{M}_{f i}^{(b)}+\mathcal{M}_{f i}^{(b r e m)}= \\
=\frac{\mathcal{A}}{q^{2}}\left[\frac{a\left(q^{2}, k^{2}\right)}{M_{1}} \varepsilon_{\mu v k q}-i M_{1} b\left(q^{2}, k^{2}\right) g_{\mu v}+2 i \frac{c\left(q^{2}, k^{2}\right)}{M_{1}} q_{\mu} k_{v}\right] j^{v}\left(k_{2}, k_{1}\right) J^{\mu}\left(k_{4}, k_{3}\right) .
\end{gathered}
$$

The $B^{-} \rightarrow \ell^{+} \ell^{-} \bar{v}_{\ell^{\prime}} \ell^{\prime-}$ differential branching ratio can be calculated by the formula

$$
d \operatorname{Br}\left(B^{-} \rightarrow \ell^{+} \ell^{-} \bar{v}_{\ell^{\prime}} \ell^{\prime-}\right)=\tau_{B^{-}} \frac{\sum_{s_{1}, s_{2}, s_{3}, s_{4}}\left|\mathcal{M}_{f i}^{(1234)}\right|^{2}}{2 M_{1}} d \Phi_{4}^{(1234)}
$$

where $\tau_{B^{-}}$is the $B^{-}-$meson lifetime, $d \Phi_{4}^{(1234)}$ - the four-body phase space, and summation is performed over the spins of final-state fermions. A more detailed theoretical description of the kinematic parameters and amplitude of four-lepton decays can be found here [5]. 


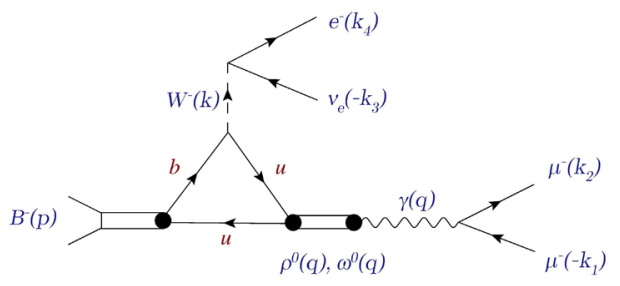

a)

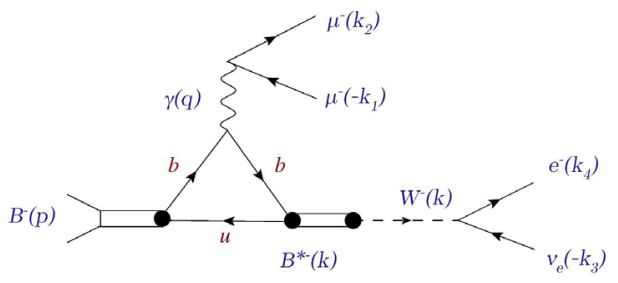

b)

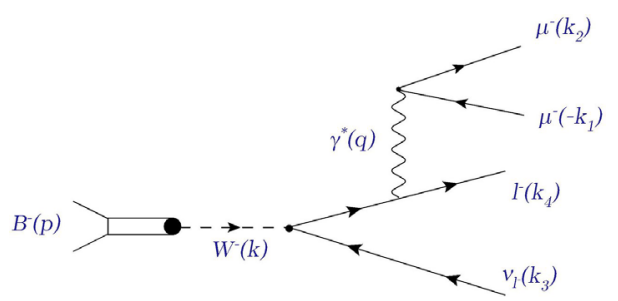

c)

Figure 1. a) Diagram corresponding to virtual-photon emission from the light quark $B-$ meson; b) Diagram involving the virtual photon emission from the heavy quark of the $B$ - meson; c) Diagram corresponding to virtual-photon bremsstrahlung.

\section{Numerical results for the branching ratio and differential distributions of the decay $B^{-} \rightarrow \mu^{+} \mu^{-} e^{-} \bar{v}_{e}$}

\subsection{Theoretical prediction}

In this section we present our numerical results for the decays $B^{-} \rightarrow \mu^{+} \mu^{-} e^{-} \bar{v}_{e}$. After the calculation of the square of the matrix element and integration over the squared momentum of the lepton pair variables $x_{12}=q^{2} / M_{1}^{2}$ and $x_{34}=k^{2} / M_{1}^{2}$, we got the numerical prediction for the $B^{-} \rightarrow \mu^{+} \mu^{-} e^{-} \bar{v}_{e}$ branching ratio:

$$
\operatorname{Br}\left(B^{-} \rightarrow \mu^{+} \mu^{-} e^{-} \bar{v}_{e}\right) \approx 1,3 * 10^{-7} .
$$

Let us mention that all leptons are taken massless in the calculations of the matrix element, but the integration startes from double muon mass. In order to avoid the divergence related to the photon propagator. The integration cut off lower bound is taken at the at double muon mass: $x_{12}^{\min }=\left(2 m_{\mu} / M_{1}\right)^{2} \approx 0.0016$.

From the experimental point of view for the $\sqrt{q_{\text {min }}^{2}}>2 m_{\mu}$ it shall be observed the same value for the branching ration of the $B^{-} \rightarrow e^{+} e^{-} \bar{v}_{\mu} \mu^{-}$decay. And the measuring and comparing branching ratios of these decays may be a test of lepton universality [5].

The differential distribution for decay rates are shown in Fig. 2, where we can seen a pole corresponding to virtual photon emission and a contributions from the $\rho^{0}(770)$ and $\omega(782)$ on the $x_{12}-$ distribution. And the $x_{34}-$ channel does not include any resonances, and we obtain a smooth function.

\subsection{EvtGen model for rare four-leptonic B-decays}

In this work we prepare the new Monte Carlo model for rare four-leptonic B-mesons decays based on the EvtGen generator package [6] BUTOMMENU. In the framework of our new 

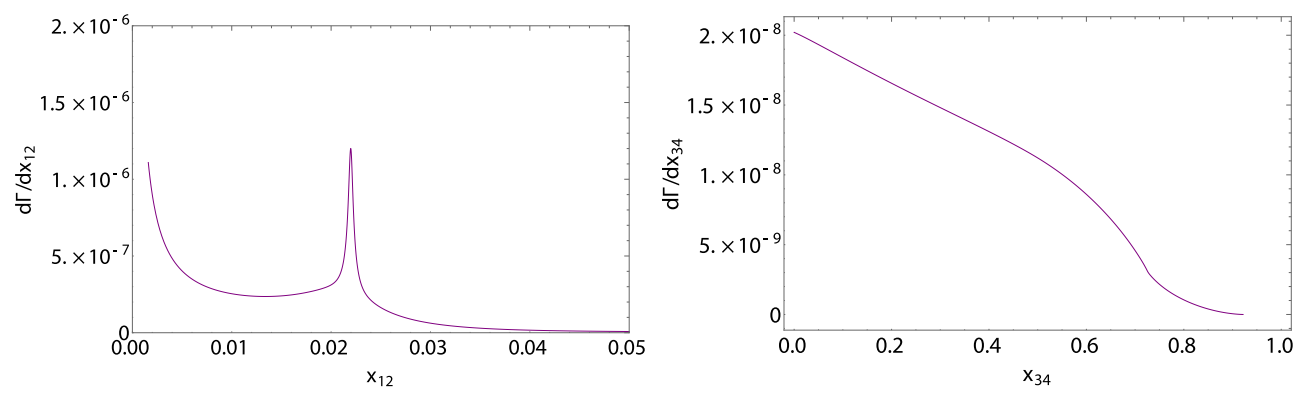

Figure 2. The $x_{12}-\left(\right.$ left), $x_{34}-$ (right) distributions for the $B^{-} \rightarrow \mu^{+} \mu^{-} e^{-} \bar{v}_{e}$ decay.

BUTOMMENU model the relativistic quark model for transition form factors calculations is used. In addition, the contributions of the $\rho^{0}(770)$ and $\omega(782)$ resonances are included. In the Fig. 3 we present out the result for the invariant dimuon mass distribution $q^{2}$ and the $k^{2}-$ distribution getting in the framework of the BUTOMMENU model. Since in different models there is different number of events contributing into the $q^{2}$ - and the $k^{2}$ - distributions, it's ok to normalize all the histograms to unity. In this model we consider the rest frame of $B$-meson and it is possible to compare with previous result. And you can seen a good agreement with result shown in the Sect. 3.1, where the same distributions were obtained by WM package (see Fig. 2). The differences on the $q^{2}$ - distribution result from the cuts at EvtGen generator level.
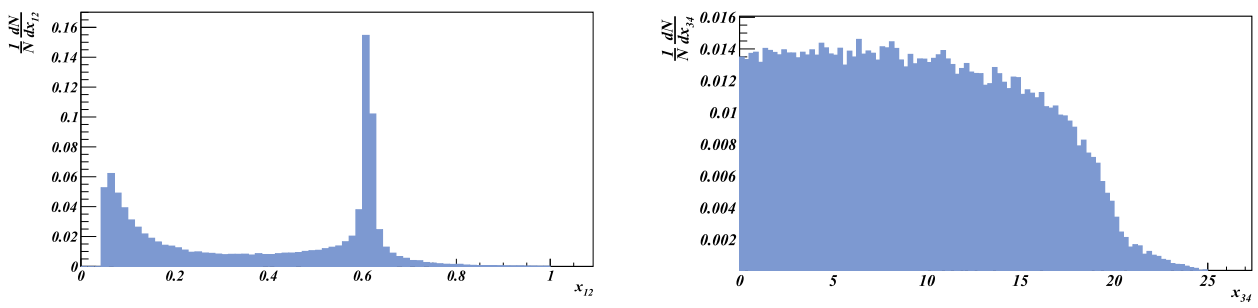

Figure 3. The $x_{12}=q^{2} / M_{1}^{2}$ - (left) and $x_{34}=k^{2} / M_{1}^{2}$ - (right) distributions for the $B^{-} \rightarrow \mu^{+} \mu^{-} e^{-} \bar{v}_{e}$ decay obtained by BUTOMMENU model.

\subsection{Distributions for the Forward-Backward Asymmetry}

The Forward-Backward Asymmetry in the $q^{2}-$ channel $A_{F B}\left(q^{2}\right)$ for the $B^{-} \rightarrow \mu^{+} \mu^{-} e^{-} \bar{v}_{e}$ decay is given by:

$$
A_{F B}^{\left(B^{-}\right)}\left(q^{2}\right)=\frac{\int_{0}^{1} d \cos \theta \frac{d^{2} \Gamma\left(B^{-} \rightarrow \mu^{+} \mu^{-} e^{-} \bar{v}_{e}\right)}{d q^{2} d \cos \theta}-\int_{-1}^{0} d \cos \theta \frac{d^{2} \Gamma\left(B^{-} \rightarrow \mu^{+} \mu^{-} e^{-} \bar{v}_{e}\right)}{d q^{2} d \cos \theta}}{\frac{d \Gamma\left(B^{-} \rightarrow \mu^{+} \mu^{-} e^{-} \bar{v}_{e}\right)}{d q^{2}}},
$$

where $\theta$ is the angle between the direction of $\ell^{\prime-}-$ lepton and direction of $B$ - meson in the rest frame of $\ell^{\prime} \bar{v}_{\ell^{\prime}}$ pair. The same definition may be written for the $B^{+} \rightarrow \mu^{-} \mu^{+} e^{+} v_{e}$ decay 
and it is obvious, that

$$
A_{F B}^{\left(B^{-}\right)}\left(q^{2}\right)=-A_{F B}^{\left(B^{+}\right)}\left(q^{2}\right) .
$$
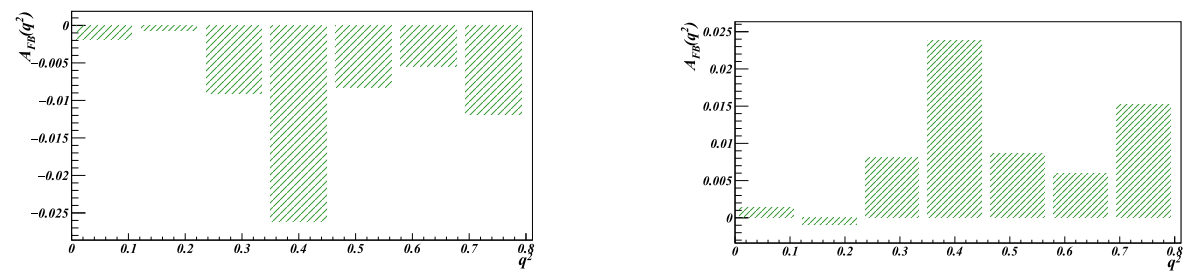

Figure 4. The $A_{F B}\left(q^{2}\right)$ - distributions from the BTOMUMUENU for the $B^{-} \rightarrow \mu^{+} \mu^{-} e^{-} \overline{v_{e}}$ (left) and $B^{+} \rightarrow \mu^{-} \mu^{+} e^{+} v_{e}$ (right) decays.

In the Fig. 4 present out results for the distributions of the forward-backward asymmetry for $B^{+} \rightarrow \mu^{-} \mu^{+} e^{+} v_{e}$ and $B^{-} \rightarrow \mu^{+} \mu^{-} e^{-} \bar{v}$ decays. In the $q^{2}-$ channel the electromagnetic interaction prevails and $A_{F B}\left(q^{2}\right)$ is close to zero. Deviation the $A_{F B}\left(q^{2}\right)$ from zero in this channel may be indicate as new parity-violating interactions beyond the Standard Model.
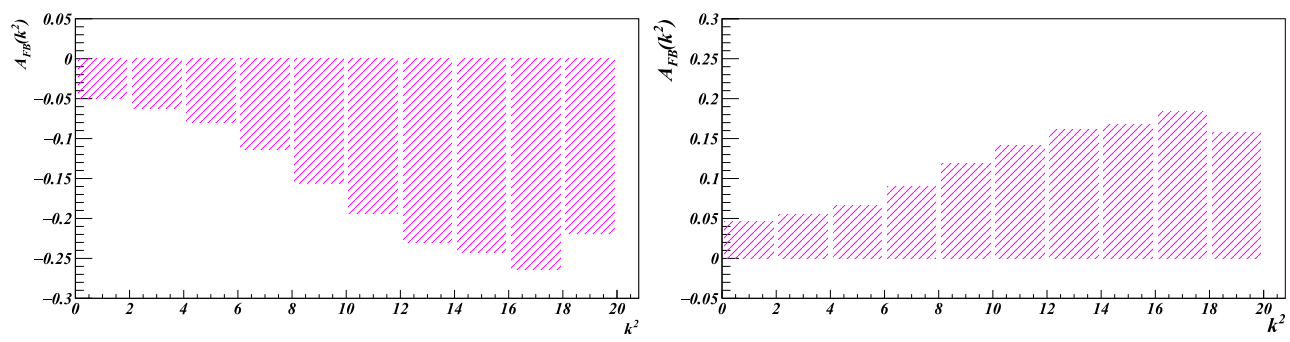

Figure 5. The $A_{F B}\left(k^{2}\right)$ - distributions from the BTOMUMUENU for the $B^{-} \rightarrow \mu^{+} \mu^{-} e^{-} \bar{v}_{e}($ left $)$ and $B^{+} \rightarrow \mu^{-} \mu^{+} e^{+} v_{e}$ (right) decays.

In the $k^{2}$ - channel we define the angle $\theta$ as angle between the direction of $\ell^{-}-$lepton and the direction of $B$ - meson in the rest frame of $\ell^{-} \ell^{+}$- pair. For the $k^{2}$ - channel (see Fig. 5) the weak contribution is dominant, and in this case the Forward-Backward Asymmetry is large and negative for the $B^{-}$- decay and large and positive for the $B^{+}$- decay within the Standard Model. But as in previous instance,

$$
A_{F B}^{\left(B^{-}\right)}\left(k^{2}\right)=-A_{F B}^{\left(B^{+}\right)}\left(k^{2}\right) \text {. }
$$

\section{Numerical results for the branching ratio of the decay $B^{-} \rightarrow \mu^{+} \mu^{-} \mu^{-} \bar{v}_{\mu}$}

From the experimental point of view, the decay $B^{-} \rightarrow \mu^{+} \mu^{-} \mu^{-} \bar{v}_{\mu}$ is of great interest to the detection. In this decay, the final state involves two identical negatively charged muons. This implied the need to take into account Fermi's antisymmetry. As described in [5], the total $B^{-} \rightarrow \mu^{+} \mu^{-} \mu^{-} \bar{v}_{\mu}$ decay amplitude has the form

$$
\mathcal{M}_{f i}^{(t o t)}=\mathcal{M}_{f i}^{(1234)}-\mathcal{M}_{f i}^{(1432)}
$$


where $\mathcal{M}_{f i}^{(1234)}$ is given by (7), while the amplitude $\mathcal{M}_{f i}^{(1432)}$ is obtained from $\mathcal{M}_{f i}^{(1234)}$ with replacement $k_{2} \leftrightarrow k_{4}$. For the respective branching ratio we have

$$
\operatorname{Br}\left(B^{-} \rightarrow \ell^{+} \bar{v}_{\ell} \ell^{-} \ell^{-}\right)=\operatorname{Br}\left(B^{-} \rightarrow \ell^{+} \ell^{-} \bar{v}_{\ell^{\prime}} \ell^{\prime-}\right)-\operatorname{Br}_{\text {interf }}\left(B^{-} \rightarrow \ell^{+} \bar{v}_{\ell} \ell^{-} \ell^{-}\right),
$$

where

$$
\begin{aligned}
\operatorname{Br}_{\text {interf }}\left(B^{-}\right. & \left.\rightarrow \ell^{+} \bar{v}_{\ell} \ell^{-} \ell^{-}\right)= \\
& =\frac{\tau_{B^{-}}}{4 M_{1}} \int \sum_{s_{1}, s_{2}, s_{3}, s_{4}}\left(\mathcal{M}_{f i}^{(1234) \dagger} \mathcal{M}_{f i}^{(1432)}+\mathcal{M}_{f i}^{(1432) \dagger} \mathcal{M}_{f i}^{(1234)}\right) d \Phi_{4}^{(1234)} .
\end{aligned}
$$

From the numerical integration of expression (11) at $x_{12 \min }=\left(2 m_{\mu} / M_{1}\right)^{2}$ were obtained

$$
\operatorname{Br}_{\text {interf }}\left(B^{-} \rightarrow \mu^{+} \bar{v}_{\mu} \mu^{-} \mu^{-}\right) \approx-2.1 \times 10^{-9}
$$

This value is one order of magnitude smaller than the uncertainty associated with the nonperturbative strong interaction effects contributions in this decay. Thus, in the limit of zero lepton masses, from (10) it can be concluded that

$$
\operatorname{Br}\left(B^{-} \rightarrow \mu^{+} \mu^{-} \mu^{-} \bar{v}_{\mu}\right) \approx 1.3 \times 10^{-7} .
$$

\section{Conclusion}

We presented predictions the branching ratios and the differential distributions for the decays $B^{-} \rightarrow \mu^{+} \mu^{-} e^{-} \bar{v}_{e}$ and $B^{-} \rightarrow \mu^{+} \mu^{-} \mu^{-} \bar{v}_{\mu}$. We took into account the processes of the photon emission from $u$ - and $b$-quarks of the $B$ - meson and the bremsstrahlung. The following predictions are obtained:

$$
\operatorname{Br}\left(B^{-} \rightarrow \mu^{+} \mu^{-} e^{-} \bar{v}_{e}\right) \approx B r\left(B^{-} \rightarrow \mu^{+} \mu^{-} \mu^{-} \bar{v}_{\mu}\right) \approx 1.3 \times 10^{-7}
$$

We prepare the new Monte Carlo model BUTOMMENU based on the EvtGen generator package for rare four-leptonic decays of charged B-mesons. Using this model we obtained distributions for the branching fractions and the forward backward asymmetry on the different lepton pair channels. We obtain a good agreement between theoretical prediction founded by different methods.

We would like to express our deep gratitude to D. Melikhov and A. Kozachuk for multiple helpful discussions which improved the paper significantly. A.Danilina is grateful to the "Basis" foundation for a stipendium for PhD students. Sections 3.2, 3.3 and 4 were done with support of the "Basis" foundation stipendium for PhD students. Sections 1,2 and 3.1 were done with the support of Grant No. 16-12-10280 of the Russian Science Foundation (A. D. and N. N.). The authors express their sincere gratitude for this support.

\section{References}

[1] V. Khachatryan et al. [CMS and LHCb Collaborations], Nature 522, 68 (2015).

[2] M. Aaboud et al. [ATLAS Collaboration], Eur. Phys. J. C 76, no. 9, 513 (2016).

[3] R. Aaij et al. [LHCb Collaboration], Phys. Rev. Lett. 118, no. 19, 191801 (2017).

[4] A. Danilina, N. Nikitin, EPJ Web Conf. 158, 03005 (2017).

[5] A. Danilina, N. Nikitin, Physics of Atomic Nuclei 81 no. 3, 331 (2018).

[6] A. Ryd et al.,"EvtGen. A Monte Carlo Generator for B-physics", on web-page https://evtgen.hepforge.org.

[7] D. Melikhov, N. Nikitin, K. Toms, Physics of Atomic Nuclei 68, 1842 (2005).

[8] D. Melikhov and B. Stech, Phys. Rev. D 62, 014006 (2000).

[9] D. Melikhov, Phys. Rev. D 53, 2460 (1996).

[10] D. Melikhov, Phys. Rev. D 56, 7089 (1997). 